\title{
Research on Library Innovation Service in Micro-era
}

\author{
Wang Zhigang \\ Yun Yang Teachers' College \\ Shi Yan, Hu Bei, 442200 China
}

\begin{abstract}
At present, various new mediarepresented bymicroblog and wechat has lead us into the mirco-era. This paper gives the connotation of micro era concept and its characteristics, and then gives an analysis of library service innovation in micro-era, such as construction and micro group application, wechat information services, micro reading adaptation and guidance, micro book reviews and so on in order to provide reference for the related researchers.
\end{abstract}

Keywords- micro-era; library; innovation service

\section{CONCEPT OF MICRO-ERA}

At present, with the continuous development and application of time and technology, various new media gradually appear in our view, applications of micro era and people's life has been closely linked together. Popularization of micro-blog makes the "micro era" quickly ascends the stage of the Internet. The emergence of micro micro-blog opened new era, then a series of micro things followed, and gradually changing the way of thought and behavior in today's era of people. Concept of "micro era", a general definition is this: "micro era" refers to the micro blog, wechat public platform as a media representative to short refining as culture transmission characteristics of the times. Since the micro Blog has been born, more and more new things are named to "micro" title. From the "micro era" contains the content, it contains the application of micro Bo, micro letter related micro platform, including micro fiction, micro quotations, micro film, micro public welfare, micro travel etc.

In the micro era, everyone can create their own micro quotations, each people all are in one record, can be a reporter can also be edited, can speak freely, and hold more discourse right. In the micro era, the dissemination of information has gotten rid of the gradual, top-down propagation mode, presents the structure mesh, geometry fission type. Micro blog, wechat have add buddy function, the user may select the character and the specific domain according to their interests, the spontaneous formation of small group. They can at any time publish and receive information, and the information is completely open to the public, can be searched. "The speed of information dissemination of micro era is fast and high efficiency. In the process of information communication, the communicator, information reduction makes the information dissemination content update more quickly.

\section{LIBRARY INNOVATION SERVICE IN MICRO-ERA}

\section{A. Innovation Service in Micro-blog}

In the micro era, the library micro-blog are mostly comprehensive accounts, which subject classification are confusion, content is multifarious, user information inconvenience, affect the degree of attention. So it is necessary to set up a plurality of Library department account, micro-blog system is conducive to the construction of library, library and readers and classification of communication between various departments of communication and coordination. According to the different service content and scope, the actual work with reference to the existing library department needs, can set up a document service micro-blog reference Exhibition Lecture, resources construction and the characteristic collection is divided into account micro-blog system construction of library. Various departments with full name and account the best library through certification head with KCBS, badge or library building, the background for this museum can also select picture, elegant picture, the overall style is consistent. The official account need to drive the sector trumpet. Choose "attention", strengthen interaction, forwarding comments, account and library between microblog, and expand the coverage of the whole information. However, the real advantage of cloud computing in this remote access is mainly reflected in the following aspects: first, the cloud data must be processed using standard metadata format digital information on a variety of sources, in order to create digital content can be retrieved; secondly, the use of standard model and tool enables developers to ignore the number format information. Develop standardized information retrieval system. Therefore, based on open standards, digital information created standard will greatly contribute to the development of information retrieval, including the data content identification, index and specific data used to create domain specific tool for mining use.

The library should establish the blog group, will have the same hobby and the characteristics of readers together, providing around a certain theme discussion and exchange of places, such as Chinese, English, marketing, etc.. Each group is in the charge of an academic subject librarian, invite industry experts to guide. Implementation of personalized management, and strengthen the interaction. On the one hand, enrich the content of services, on the other hand to build understanding and listen to the needs of the readers' channel. We can also be combined with expert system and library micro-blog to the library website as a platform for the creation of "virtual learning community" and "virtual reference" and "virtual subject navigation" and other services. 


\section{B. Innovation Service in Wechat}

Wechat is launched by Tencent Inc in 2011, can be text, voice, picture and video communication in the intelligent mobile phone and so on, providing people chat and $1 \mathrm{~km}$ within the user search, otherwise shake function and drift bottle and dating, currently has over 400 million users. The library information servicebased on the huge user groups develops the following modes.

Wechat is no doubt more advantages by combining with LBSin improving library service concern the target population positioning of the nearby library or university, can be one to one active greetings. Friendly ways make users feel the library concern and respect. Not limited to text, but also through the video, pictures, voice and other forms of graffiti, instant close distance. Let the user feel is in making friends, rather than work, more easy to accept service.

Wechat users search the library account online consulting in case of doubt in the library to borrow, or in the study are in urgent need of reference materials. The librarian immediately retrieval information and timely feedbackafter receivereceipt of a request, Reply to the consultation can be text, voice, video to explain, can be directly screenshots. Different forms, diverse portfolio, more vivid.In addition to a passive response can also be active service first search to establish user social networks provide. That is, by searching the QQ and address book friends, to see the crowd and drift bottle, inside 1 kilometer shake etc., micro credit account L search. Request add buddy, the establishment of micro believe friends circle. And then take the initiative to provide all kinds of information services, such as library news, announcements, new book notification and new database information. Whether public or university libraries, we can with the subject characteristics in micro letter development librarian service. As announced on the website micro letter number, allow users to contact with friends. We can also take the initiative to collect subject users, such as university library through professional class teaching and research section, micro letter acquiring the number of students and teachers. Because the information is free, voice communication and other advantages, the reader is easy and subject librarian to establish long-term relationship. Subject librarians can achieve communication without the interview with the users. As readers of the field study or travel, can also through the micro letter to get free information support. The communication characteristics of micro letter determine it's more attractive than telephone, QQ and mailbox. Narrow the distance between the two sides carry out to service.

\section{Innovation Service in Micro-reading}

On one hand, the micro reading environment is a huge information resource, but on the other hand it is the reading of the superficial and debris. This brings new challenges to the library service, not only to adapt to the trend, enrich service content and form; and the need to provide active guidance, knowledge resources comprehensive system, improve the reader deep reading ability.

The characteristics of micro reading of university library resource database and usually have all kinds of professional journals and other rich. We can provide academic and professional micro reading. The public library can focus on the national reading and the popularization of culture and knowledge.By gender and age registered information largely determines the reading tendency. According to books borrowing history, electronic resources such as the use of records, can be further analyzed its habits. University library can also through the student's professional judgment and grade. As undergraduates usually focus on the new book information and electronic resources update, and graduate students and teachers on their own whether the article by SCI and EI included and related conference information such as a higher degree of concern.

On the one hand, the library for some hot issues and important information through the micro reading platform to publish timely push reports, enable the reader to understand the first time; on the other hand, micro reading platform feedback and suggestions by readers can, create a good interactive atmosphere.

Tracking and manufacturing hotspot in current event hot books and periodicals and electronic resources etc., the timely introduction of the corresponding micro reading content. In the manufacture of hot spots, you can learn from the Internet marketing ideas, targeted publicity to attract reader's attention.

\section{Innovation Service in Micro Book Reviews}

Micro book review is a new text form and propagation behavior, usually no more than 140 Chinese characters. From 2010 under the banyan "first micro book review contest", to dangdang.com and Tencent jointly launched the "China micro review, set off a" micro era of reading interaction climax. As a new service mode of the library, in brief, insight language to recommend books, a bridge between construction and reader, can realize the navigation information, but also can increase the virtual collection, and promote the relative academic research.

As a part of the library service micro book review should take the readers as the starting point, the integration of library resources and human resources development, conduct propaganda. On the literature database, electronic publications and Internet information, preparing and refining, mining, provide to the different needs of the readers. First of all, we can hire experts as advisers, to strengthen the academic research. Secondly, taking the library of human resources as the backbone, play the advantage of subject librarian, professional division of labor to write. It may also be involved in the absorption, certain professional knowledge literacy readers to strengthen training, encourage writing. At the same time, we establish the Micro Book Reviews Association to make plan or organize activities step by step.

\section{CONCLUSION}

The things generated in the micro era background have grown to promote the reading patterns and change our life mods. The library's innovative service will be more and more important. We can serve the readers better through the micro-blog, wechat, micro reading and micro book reviews and so on. 


\section{REFERENCES}

[1] Guan Xin. Discussion on the Application of Micro-blogging in University Libraries [J].Journal of Library and Information Sciences in Agriculture, 2012, 05
[2] Xiao Juan. Micro-blog Application Status and Analysis in our Library_-Based on the Sina micro-blog [J]Guizhou Library Publication.2011, 02 